\title{
RESULTADOS PRELIMINARES DEL ESTUDIO DE LA CALIDAD DE LA CANAL DE LA RAZA BOVINA MALLORQUINA
}

\author{
PRELIMINARY RESULTS OF THE STUDY OF THE CARCASS QUALITY OF \\ THE MAJORCAN CATTLE BREED
}

\author{
Nogales, S. ${ }^{1 *}$, Puigserver, G. ${ }^{2 A}$, Crespi, J.L. ${ }^{2}$, Pons, A. ${ }^{3}$, Delgado, J.V. ${ }^{1 A}$ y Camacho, M.E. ${ }^{4}$ \\ 'Departamento de Genética. Universidad de Córdoba. Córdoba. España. *seio21@hotmail.com; \\ Ajuanviagr218@gmail.com \\ ${ }^{2}$ Associació de Criadors de Bestiar Boví de Raça Mallorquina. Artà. Mallorca. España. \\ Agabrielpuigserver@gmail.com \\ ${ }^{3}$ Instituto de Biología Animal de Balears. Sineu. Mallorca. España. rraa@ibabsa.net \\ ${ }^{4}$ IFAPA. Alameda del Obispo. Consejería de Innovación, Ciencia y Empresa. Córdoba. España. \\ mariae.camacho@juntadeandalucia.es
}

\section{Palabras clave adicionales}

Productos locales. Caracterización de producciones. Conservación.

\section{RESUMEN}

La raza bovina Mallorquina, tras haber estado muy cerca de la extinción, se encuentra en plena recuperación a causa de la puesta en valor de sus productos cárnicos, los cuales se comercializan de forma muy restringida con un alto valor agregado. Se hizo necesario caracterizar dichos productos para protegerlos de fraudes o imitaciones que pudieran poner en riesgo su credibilidad en el consumidor. En el presente estudio se aportan los resultados preliminares de la caracterización de la canal del producto más considerado de la raza, la carne de bou. Se estudiaron las características de las canales de 7 toros de la raza como parte del trabajo de caracterización productiva. Los animales sacrificados fueron machos sin castrar con una edad media en torno a los 32 meses de edad al sacrificio estudiándose los pesos, rendimientos y morfometría, así como el pH y la clasificación comercial de la canal. Al despiece se calcularon los porcentajes de composición de la canal en hueso, grasa y carne, y se realizó un seguimiento del despiece comercial. Las canales producidas son pesadas de buen rendimiento de la canal, bajo contenido en grasa y alto en músculo y hueso. Se trata de un producto totalmente diferenciable y protegible bajo una marca de calidad.

Presentado al Congreso SERGA (2010, Asturias).

\section{AdDitiOnAL KEYWORDS}

Local products. Characterization of productions. Conservation.

\section{SUMMARY}

Mallorquina cattle breed, after a period of extreme endangering, is in a recuperation process because of the valuation of their meat products; they are commercialized in a restricted way with a high added value. The characterization of these products became necessary to protect them from frauds and imitations which could put in risk its credibility in the consumer. In the present study preliminary results on the characterization of the most valuated product of the breed, the bou meat, are presented. Carcass characteristics of 7 bulls of the breed were studied as a part of the productive characterization of the breed. Animals were no castrated males slaughtered with a mean age around 32 months. Weights, yields and morphometry were studied in the carcasses, together with $\mathrm{pH}$ and commercial classifications. After commercial cutting, the bone, fat and muscle composition of the carcasses were determined. Carcasses obtained were heavy with good carcass yields, low fat contents and high in bone and muscle. It is a product easily to differentiate and protect under a quality trade mark.

\section{INTRODUCCIÓN}

El bovino Mallorquín ha formado parte del paisaje y la cultura de la isla de Mallor- 
ca en el pasado, hasta que en el anterior siglo, debido a los cambios sociales producidos, estuvo cercano a la extinción. Entre otras causas, la revalorización de otros productos agrarios y la eliminación de los animales en las labores de labranza, añadido a la presión del turismo y al impacto de la globalización provocaron esta situación. Hoy en día, la creciente preocupación por un sector de la población mundial por encontrar formas de producción sustentables y en equilibrio con el medio ambiente, sumado a la preservación de la cultura tradicional y el patrimonio histórico, han dado sentido a que se tomen las medidas oportunas para que esta raza bovina siga existiendo. Así pues, en los años 80 comienza el proceso de recuperación (Puigserver y Lliteres, 2000). Actualmente, uno de los objetivos es caracterizar la producción cárnica de la raza, de forma que contribuya a mantener el valor añadido de la carne y mejore la preservación de este recurso zoogenético oficialmente reconocido Real Decreto 2129/2008 del MMAMRM. En el presente trabajo se adelantan los resultados preliminares de la caracterización de la canal de la raza.

\section{MATERIAL YMÉTODOS}

Se emplearon 7 animales machos enteros de la raza Mallorquina con una edad al sacrificio aproximada de 32 meses. Los animales provenían del cebadero de la asociación de criadores de la raza, donde fueron alimentados con una mezcla de harina de cebada y habas, y como porción fibrosa paja de cebada y gavilla de avena, proviniendo todos los alimentos de cultivos de la isla de Mallorca. Faenada la canal se obtuvieron los datos proporcionados por personal de matadero sobre la clasificación de la canal (Reglamento UE N ${ }^{\circ} 1183$ / 2006) según escala SEUROP, a la cual se le asignaron valores del 1 al $15(\mathrm{P}=2, \ldots$, $\mathrm{E}=14)$ y el estado de engrasamiento, con escala del 1 al $9(1=$ magra $, \ldots, 9=$ muy grasa $)$
(Zea et al., 2007b). A continuación, se tomaron las medidas morfológicas en la canal propuestas por De Boer et al. (1974): longitud total de la canal (LTC), profundidad interna del pecho (PIP), longitud de la pierna (LP) y perímetro máximo de la pierna (PMP). A partir del peso vivo al sacrificio (PV) y el peso de la canal madurada (PCM), se determinó el rendimiento comercial como $\mathrm{RC}=\left(\mathrm{PCM}^{*} 100\right) / \mathrm{PV}$. Igualmente se estimaron las pérdidas porcentuales en la canal durante el proceso de maduración como: [(Peso canal caliente - Peso canal madurada)*100]/Peso canal caliente. También fue calculado el Índice de Compacidad, ICC= PCM/LTC. El pH se determinó con un pHmetro portátil $\mathrm{pH} 25$ (Crison Instruments, S.A., Barcelona, España), midiéndose al sacrificio, a las 24 horas, y una vez terminado el proceso de la maduración. La maduración de la canal fue realizada en las cámaras del matadero a una temperatura de entre 2 y $5^{\circ} \mathrm{C}$. El tiempo de maduración no fue el mismo para todas las canales debido a que la salida de la carne depende de las variaciones del mercado, la media fue de 14,57 $\pm 1,90$ días. Al despiece fueron pesadas cada una de las piezas, y se separaron para su posterior pesada los huesos y recortes de grasa. Con la información obtenida se calcularon los porcentajes en la canal de carne de cada categoría, como peso de las piezas de la

Tabla I. Valores medios y desviaciones estándar de las variables medidas sobre conformación y morfometría. (Means and standard deviations values of the measured variables on conformation and morphometry).

\begin{tabular}{lcc}
\hline Variable & Media & DE \\
\hline SEUROP & 4,57 & 1,13 \\
Estado de engrasamiento & 4,43 & 0,98 \\
Longitud total de la canal & 127,67 & 7,39 \\
Profundidad interna del pecho & 44,42 & 3,04 \\
Longitud de la pierna & 75,33 & 4,46 \\
Perímetro máximo de la pierna & 106,75 & 15,98 \\
Índice de compacidad & 1,86 & 0,19 \\
\hline
\end{tabular}




\section{CALIDAD DE LA CANAL DE LARAZA BOVINA MALLORQUINA}

Tabla II. Valores medios y desviaciones estándar del pH al sacrificio, a las 24 horas y al final de la maduración, medidos en el Longissimus thoracis. (Means and standard deviations values for the $\mathrm{pH}$ at slaughtering, at 24 hours and at the end of the ageing process, measured in Longissimus thoracis).

\begin{tabular}{cccccc}
\hline \multicolumn{2}{c}{ Sacrificio } & \multicolumn{2}{c}{24 horas } & \multicolumn{2}{c}{ Final maduración } \\
media & $D E$ & media & DE & media & DE \\
6,88 & 0,15 & 5,68 & 0,42 & 5,56 & 0,14
\end{tabular}

categoría * 100/PCM. Para el análisis de los datos se han utilizado los cálculos de la media y las desviaciones estándar.

\section{RESULTADOSYDISCUSIÓN}

El primer dato a destacar como poco frecuente en el mercado español fue el peso vivo medio al sacrificio situado en los $445 \pm 17,32 \mathrm{~kg}$. En la tabla I se presentan los valores sobre conformación. Se observa que estas canales se hallan, en su mayoría, clasificadas como "O" (menos buena), mientras que las canales de otras razas españolas obtienen mejor valoración según esta clasificación (Panea et al., 2010 en Menorquina; Zea et al., 2007a en Rubia Gallega). Al estudiar el estado de engrasamiento se aprecia que las canales presentan una cobertura grasa escasa. Las variables medidas en la morfología de la canal no varían en gran medida con las de otras razas consultadas en la bibliografía (Albertí et al., 2005). El índice de compacidad hallado, de 1,86, también se muestra inferior al de otras razas estudiadas en España, como en el caso de la Retinta (Alberti et al., 1995). Por otro lado, en la tabla II se presentan las medidas de $\mathrm{pH}$, incluido el momento final de maduración. Se observa la caída del $\mathrm{pH}$, que se mantiene en el rango normal para la comercialización de la carne de vacuno (Tarrant y Sherington, 1980). En lo que respecta al rendimiento comercial, la Mallorquina ofrece un valor bastante positivo $(54,57 \%)$ que adquiere mayor interés al estudiarlo conjuntamente con el porcentaje de carne $(74,87 \%)$, el cual muestra unos valores medios-altos respecto a la bibliografía (Paena et al., 2010; Zea et al., 2007b). A lo anterior hay que sumar la maduración que representa unas pérdidas en la canal del $3,25 \%$, cuando se estima que en las primeras 24 horas la canal sufre pérdidas aproximadas de un $2 \%$, aunque variando sustancialmente entre los animales hasta ahora estudiados. En la composición de la canal también es reseñable el porcentaje de hueso, que puede parecer alto, pero se ha de tener en cuenta que el bajo valor de la grasa que se promedia en un $3,65 \%$, y difiere en gran medida con otras razas (Cañeque y Sañudo, 2000). En la tabla III se encuentra representada la categorización de las piezas de la canal, destacando el alto porcentaje de piezas de segunda categoría. Esto se debe a variaciones en la categorización de las piezas respecto a la península, pues hay un porcentaje significativo de carne que se destina para estofado y en consecuencia se categoriza como de segunda para la comercialización.

Tabla III. Valores medios y desviaciones estándar de las variables estudiadas sobre el rendimiento y composición de la canal. (Means and standard deviations values of the measured variables on carcass yield and composition).

\begin{tabular}{lcr}
\hline Variables & media & \multicolumn{1}{c}{ DE } \\
Peso vivo al sacrificio & 445 & 17,32 \\
Peso canal madurada (CM) & 235,47 & 22,79 \\
Rendimiento comercial \% (CM) & 54,57 & 4,12 \\
Pérdidas por maduración \% & 3,25 & 0,91 \\
Carne \% & 74,87 & 1,22 \\
Hueso \% & 18,66 & 1,92 \\
Grasa \% & 3,65 & 0,80 \\
Categorización de las piezas comerciales & \\
Solomillo & 1,94 & 0,13 \\
Lomo & 9,93 & 0,44 \\
Primera & 38,08 & 1,79 \\
Segunda & 19,11 & 1,06 \\
Tercera & 5,37 & 0,96 \\
\hline
\end{tabular}




\section{NOGALES, PUIGSERVER, CRESPI, PONS, DELGADOY CAMACHO}

\section{CONCLUSIONES}

La canal de machos enteros de raza Mallorquina sacrificados a 32 meses y alimentados con pienso se caracteriza por ser una canal pesada, de baja calificación convencional, que muestra un buen rendimiento general y de piezas nobles, resaltando aquí que se valoran en el mercado hasta las piezas de segunda debido a la elaboración de estofados tradicionales. Destaca un bajo contenido graso y una alta proporción de músculo y hueso, como es de esperar en

\section{BIBLIOGRAFÍA}

Alberti Lasalle, P., Sañudo Astiz, C., Santolaria Blasco, P., Lahoz Castelló, F., Olleta Castañer, J.L. y Campo Arribase, M.M. 1995. Características de la canal y calidad de la carne de añojos de la raza Retinta. Arch. Zootec., 44: 283-295.

Albertí, P., Ripoll, G., Goyache, F., Lahoz, F., Olleta, J.L., Panea, B. y Sañudo, C. 2005. Carcass characterisation of seven Spanish beef breeds slaughtered at two commercial weights. Meat Sci., 71: 514-521.

Cañeque, V. y Sañudo, C. 2000. Metodología para el estudio de la calidad de la canal y de la carne en rumiantes. Ed. Instituto Nacional de Investigación y Tecnología Agraria y Alimentaria. Ministerio de Ciencia y Tecnología. Madrid. España. $255 \mathrm{pp}$.

De Boer H., Dumont, B.L., Pomeroy, R.W. and Weniger, J.H. 1974. Manual on E.A.A.P. reference methods for the assessment of carcass characteristics in cattle. Livest. Prod. Sci., 1: 151-164.

MMAMRM. Ministerio de Medio Ambiente y Medio Rural y Marino. 2008. Real Decreto $2129 / 2008$ de 26 de diciembre, por el que se establece el animales de alta edad como los sacrificados. El estudio productivo de esta raza se va desarrollando lentamente debido al bajo número de animales que se sacrifican al año.

\section{AGRADECIMIENTOS}

Los autores desean agradecer la formidable colaboración prestada por la Dirección y todo el personal del matadero de CARN ILLA S.A. y la sala de despiece de FRIALSA, en Mercapalma, que han hecho posible el desarrollo de este estudio.

Programa Nacional de conservacion, mejora y fomento de las razas ganaderas. Boletin Oficial del Estado.

Panea, B., Sañudo, C., Olleta, J.L. y Sierra, I. 2010. Caracterización de la canal y la carne de la raza bovina Menorquina. Arch. Zootec., 59: 467-470.

Puigserver, G. y Lliteres, B. 2000. Proceso de recuperación de una agrupación racial de bovinos de Mallorca. Arch. Zootec., 49: 71-76.

Tarrant, P.V. and Sherington, J. 1980. An investigation of ultímate $\mathrm{pH}$ in the muscle of comercial beef carcasses. Meat Sci., 4: 287-297.

UE. Reglamento №1183/2006 del Consejo de 24 de julio de 2006 sobre el modelo comunitario de clasificación de las canales de vacuno pesado.

Zea, J., Díaz, M.D. y Carballo, J.A. 2007a. Efecto de la raza, sexo y alimentación en la calidad de la canal de vacuno. Arch. Zootec., 56 (Supl. 1): 745-751.

Zea Salgueiro, J., Díaz Díaz, M.D. y Carballo Santaolalla, J.A. 2007b. El efecto del sistema de producción y del sexo en el comportamiento y calidad de la canal de vacuno. Arch. Zootec., 56: 331-342. 